\title{
DISLIPIDEMIAS Y EL ACEITE SACHA INCHI
}

DISLIPIDEMIAS AND THE SACHA INCHI OIL

\author{
DISLIPIDEMIAS E O ÓLEO DE SACHA INCHI
}

\section{ALEJANDRO SUÁREZ ROBALINO \\ Universidad Católica de Santiago de Guayaquil, Guayaquil, Ecuador}

Resumen

El aumento en la incidencia de las enfermedades cardiovasculares en nuestro país toma ya aspectos alarmantes, entre cuyos detonantes se encuentran los factores de riesgo entre los cuales destacan la diabetes y las dislipidemias. Otros factores de riesgo son la hipertensión arterial, el tabaco y el sedentarismo. Entre las 10 principales causas de muerte en el Ecuador según datos del año 2008 del Instituto Nacional de Estadísticas y Censos (INEC), figuran la diabetes mellitus en 1er lugar y las enfermedades cerebrovasculares en $2^{\circ}$ lugar. Un factor de riesgo es aquel que aumenta la probabilidad en un individuo de desarrollar una enfermedad cardiovascular en un lapso de 10 anos. Por lo tanto todo fármaco que reduzca alguno de estos factores de riesgo, tendrá una incidencia positiva en las tasas de morbi-mortalidad de la población.

PALABRAS CLAVE: dislipidemia, aceite de sacha inchi, ácido araquidónico, ácidos grasos poliinsaturados omega 6 .

Abstract

The increase in the incidence of cardiovascular diseases in our country already takes alarming aspects, whose triggers are the well-known risk factors among which diabetes and dyslipidemias stand out. Other risk factors are high blood pressure, smoking and sedentary lifestyle. Among the 10 leading causes of death in Ecuador according to 2008 data from the National Institute of Statistics and Census (INEC) diabetes mellitus is in the 1st place and cerebrovascular diseases in 2nd place. A risk factor is something that increases the probability in an individual of developing a cardiovascular disease in a lapse of 10 years. Therefore, any drug that reduces any of these risk factors will have a positive impact on the morbidity and mortality rates of the population.

KEYWORDS: dyslipidemia, sacha inchi oil, arachidonic acid, omega 6 polyunsaturated fatty acids.

Resumo

0 aumento da incidência de doenças cardiovasculares em nosso país já assume aspectos alarmantes, dentre os quais estão os fatores de risco, dentre os quais se destacam o diabetes e as dislipidemias. Outros fatores de risco são pressão alta, tabagismo e sedentarismo. Entre as 10 principais causas de morte no Equador, de acordo com dados de 2008 do Instituto Nacional de Estatística e Censos (INEC) incluem diabetes mellitus em primeiro lugar e doenças cerebrovasculares em segundo lugar. Um fator de risco é aquele que aumenta a probabilidade em um indivíduo de desenvolver uma doença cardiovascular em um lapso de 10 anos. Portanto, qualquer medicamento que reduza qualquer um desses fatores de risco terá um impacto positivo nas taxas de morbidade e mortalidade da população.

PALABRAS-CHAVE: dislipidemia, óleo de sacha inchi, ácido araquidônico, ácidos graxos poliinsaturados ômega-6. 
INTRODUCCIÓN

Las Enfermedades cardiovasculares se han constituido en un azote para la población mundial. Tanto el mundo desarrollado como los países en vías de desarrollo no escapan a esta catástrofe. En nuestro país según datos del INEC del año 2003, las enfermedades cardiovasculares contribuyeron en un $22.8 \%$ a la mortalidad total, ocupando la diabetes mellitus el $3^{\circ}$ lugar con una tasa del 4.5\%. Para el 2008 la diabetes mellitus ocupaba ya el 1er puesto, con una tasa del 5.8\%.

Esto nos da una idea de cómo la diabetes y otros factores de riesgo están contribuyendo a esta elevada tasa de mortalidad en nuestro país. Por factor de riesgo cardiovascular se entiende a la condición o conjunto de condiciones que aumentan la posibilidad en un individuo de desarrollar una enfermedad cardiovascular o específicamente coronaria en un lapso de 10 años. ${ }^{1}$

Otros factores de riesgo son el colesterol y triglicéridos elevados, las cifras bajas de colesterol HDL y las altas de colesterol LDL, la hipertensión y el tabaco.

El riesgo cardiovascular se calcula de acuerdo a la escala de Framingham que asigna una puntuación a cada factor de riesgo, para establecer 3 categorías: riesgo alto con una probabilidad mayor al 20\% de sufrir un evento cardiovascular en los 10 años próximos; entre 10 y $20 \%$ para un riesgo intermedio; y menor del $10 \%$, para un riesgo cardiovascular bajo. ${ }^{2}$

Las guías médicas actuales sugieren abandonar la actitud sedentaria, incrementar la actividad física, reducir los niveles plasmáticos de colesterol LDL, asumir una dieta pobre en grasas saturadas, así como atenuar el consumo de sal. El incremento de la frecuencia cardíaca se asocia con muerte súbita; por lo tanto la reducción de esta frecuencia con una actividad física regular proporciona una mayor sobrevida. ${ }^{3}$

En esta breve revisión se habla de uno de esos factores de riesgo, las dislipidemias, y la acción que sobreéstas se ha encontrado que tiene el aceite Sacha Inchi reportada en 5 estudios.

DISLIPIDEMIAS

Se conoce por este nombre a la elevación de los niveles de colesterol y/o triglicéridos transportados por las lipoproteínas del plasma. En la práctica clínica las dislipidemias primarias corresponden un 85 a $90 \%$ de todas las dislipidemias, estando el 10 a $15 \%$ restante constituídas por dislipidemias secundarias a una enfermedad o patología de base.

CLASIFICACIÓN DISLIPIDEMIAS PRIMARIAS

Se trata de un trastorno genético o hereditario de las lipoproteínas plasmáticas.

- Hipertrigliceridemias (Fenotipo I) (Quilomicrones).

- Hipercolesterolemia (Fenotipo IIa) - LDL como fracción alterada.

- Hipercolesterolemia+ hipertrigliceridemia (Fenotipo IIb)- VLDL- LDL como fracción alterada.

- Hipercolesterolemia + hipertrigliceridemia (Fenotipo III) - IDL (Lipoproteínas de densidad intermedia).

- Hipertrigliceridemia (Fenotipo IV) - VLDL como fracción alterada.

- Hipercolesterolemia+Hipertrigliceridemia (Fenotipo V) - Quilomicrones + VLDL como fracción alterada.

\section{DISLIPIDEMIAS SECUNDARIAS}

La alteración lipoproteica se debe a una enfermedad subyacente (síndrome metabólico, diabetes, enfermedades de la tiroides, obesidad, hepatopatías, uso de fármacos, nefropatías).

\section{TRATAMIENTO DE LAS DISLIPIDEMIAS CON CAMBIOS EN EL} ESTILO DE VIDA

- Reducción de la ingesta de grasas saturadas y colesterol

- Reducción de peso

- Aumento de la actividad física

- No fumar

- Consumo moderado de alcohol (40 a $50 \mathrm{~g}$. diarios)

- Medidas dietéticas

\section{TRATAMIENTO FARMACOLÓGICO DE LAS DISLIPIDEMIAS}

- Estatinas (sinvastatina, atorvastatina, rosuvastatina)

- Fibratos (gemfibrozil, fenofibrato, bezafibrato)

- Resinas

- Inhibidores selectivos de la absorción intestinal del colesterol (ezetimibe)

- Ácido nicotínico 
- Inhibidores de la proteína que transfiere ésteres de colesterol

- Terapia hormonal con estrógenos y antioxidantes

- Ácidos grasos insaturados?

\section{LOS ÁCIDOS GRASOS INSATURADOS, LA DIETA Y LA} SALUD CARDIOVASCULAR

\section{ÁCIDOS GRASOS MONOINSATURADOS-OMEGA 9}

El ácido oleico es su representante principal. Está presente en el aceite de oliva (75\%), aceitunas, almendras, maní, y aguacates. Tambien se lo encuentra en grasas animales.

Estos ácidos llamados también omega 9 disminuyen el colesterol LDL y los triglicéridos y no afectan negativamente al colesterol HDL. El aceite de Oliva produce menor agregación de las plaquetas, reduce el factor tisular plasmático y el inhibidor tisular del plasminógeno. Estos ácidos omega 9 deberían constituir un 15\% de las calorías de una dieta diaria de 2000 calorías. El aguacate contiene un $70 \%$ de ácido oleico.

ÁCIDOS GRASOS POLIINSATURADOS-OMEGA 3-6-9

Los ácidos grasos omega 3 y omega 6 son considerados esenciales porque no son sintetizados por el organismo humano. Han demostrado reducir el colesterol total, el colesterol LDL y a diferencia de los omega 9 que actuan sobre el colesterol HDL. Sin embargo no deben constituir más del 10\% de la ingesta calórica diaria porque paradójicamente se vuelven aterogénicos al permitir la oxidación acelerada de las partículas de colesterol LDL. Tambien se aumenta el riesgo de cáncer, de cálculos biliares y de depresión del sistema inmune humano. Por esto se aconceja que su consumo sea del órden del 3 al 7\% de la ingesta calórica diaria y no pasar del $10 \%$. Esto tiene mucha importancia en patologías que aumentan el estrés oxidativo como la diabetes mellitus.

El representante más común de estos ácidos es el linoleico.

De los vegetales se obtienen el ácido linoleico (omega 6) y el alfa-linoleico (omega 3). Estos al ser consumidos por los animales quedan almacenados en sus grasas (ácido linoleico), como materia prima de reserva para producir ácido araquidónico a partir del omega 6 que queda almacenado en la carne del animal, y ácido docosaexahenoico (DHA) a partir del omega 3. Como el hombre no los sintetiza éste obtiene el ácido araquidónico de la carne de los animales. Estos dos ácidos omega deben estar en partes iguales en las membranas celulares humanas para ser beneficiosos, pero debido a la industrialización moderna de la cría de animales, esto no se da y tenemos un predominio perjudicial de omega 6 en los animales que consumimos.

Por tanto actualmente en nuestras células predominan los omega 6 sobre los omega 3. Los ácidos omega 6 en exceso se relacionan con inflamación y aterogénesis que curiosamente son los males que aquejan a la humanidad actual. ${ }^{1}$ Estos ácidos grasos esenciales son los precursores de prostaglandinas, prostaciclinas, leucotrienos y tromboxanos muchos originados a partir del ácido araquidónico. Ahora se analiza la función de algunas de estas substancias llamadas también eicosanoides.

El ácido araquidónico se convierte en prostaglandina $\mathrm{H} 2$ ( $\mathrm{PGH} 2$ ); las prostaglandinas tiene muchas funciones entre ellas participar en la inflamación y en la producción de dolor y fiebre. La PGH2 se convierte en tromboxano; el tromboxano A2 se origina en las plaquetas y favorece la vasoconstricción y agregación de las plaquetas. Las prostaglandinas son importantísimas en el ciclo reproductor femenino, en el parto, en la carcinogénesis y en la regulación de la apoptosis y la angiogénesis.

El ácido araquidónico también origina los leucotrienos los que están involucrados en la inflamación y en la alergia; inducen broncoconstricción, contraen y aumentan la permeabilidad de las arteriolas y reclutan neutrófilos y eosinófilos en los sitios de inflamación. Por lo tanto están involucrados en el asma, psoriasis, rinitis alérgica, artritis reumatoide y colitis ulcerativa. ${ }^{4}$

\section{ESTUDIOS CLINICOS REALIZADOS CON EL ACEITE VEGETAL SACHA INCHI}

Con este breve resúmen de la función y acciones en el cuerpo humano de los ácidos grasos insaturados, procede revisar brevemente también los resultados de 4 estudios realizados en el Perú y 1 realizado en nuestro país con el aceite vegetal obtenido de la planta Sacha Inchi. 
La planta sacha inchi (plukenetia volubilis linneo) pertenece a la familia euphorbiaceae y crece en la amazonía de Perú, Ecuador, Colombia y Brazil a alturas entre 200 y 1500 metros. Contiene su semilla grandes cantidades de ácido alfa-linolénico (omega 3).

Debemos conocer que en la distribución de los ácidos linoleicos -si se compara el aceite sacha inchi con el aceite de girasol- encontramos en el aceite de sacha inchi un $9.6 \%$ de omega 9 y en el de girasol un $28.9 \%$; en cuanto al omega 6 está presente en un $34.9 \%$ en el aceite de sacha inchi y en $57.2 \%$ en el aceite de girasol, y del omega 3 hay un $47.7 \%$ en el aceite de sacha inchi contra un $0.6 \%$ en el aceite de girasol. ${ }^{5}$

El estudio gemfibrozilo versus aceite de sacha inchi se realizó en ratas ( Rattus rattus var albinus) machos sanos, que fueron asignados a 2 grupos existiendo un 3er grupo control. Luego de una etapa de acondicionamiento de 2 semanas se les suministró una dieta rica en grasas a los 3 grupos durante 2 semanas obteniendo una muestra de sangre para dosificar triglicéridos al final del primer y segundo período. Después de esto al grupo 1 se le administró aceite de sacha inchi en dosis de 27 mililitros una vez al dia, y al grupo 2 una suspensión de gemfibrozilo en dosis de $3.24 \mathrm{mg}$ en 50 mililitros una vez al dia. Se tomaron muestras a la semana y 2 semanas de la administración.

Se encontró que los niveles de triglicéridos aumentaron en los 3 grupos luego de la administración de la dieta rica en grasa. Los niveles de lípidos bajaron en mayor proporción en los 2 grupos de tratamiento en comparación con el grupo control. Hubo una disminución a la semana 1 de tratamiento de $35.4 \%$, para el grupo sacha inchi; y de $34.8 \%$, para el grupo gemfibrozilo. Al final de la semana 2 la reducción de los triglicéridos para el grupo sacha inchi fue de $45.5 \%$ y de $44.8 \%$ para el grupo gemfibrozilo. Se concluyó que la disminución de los niveles de triglicéridos fue similar en ambos grupos de experimentación. ${ }^{6}$

En otro esudio efecto de la plukenetia Volubilis Linneo (Sacha inchi) en la trigliceridemia posprandial se administró a 12 individuos jóvenes entre 18 y 25 años de edad, sanos y con un IMC hasta $24.9 \mathrm{Kg} / \mathrm{m} 2$, una carga de triglicéridos consistente en 82 gramos de aceite de oliva previa toma de una muestra basal de triglicéri- dos en sangre y a las 1 1/2 horas, 3 horas, 4 1/2 horas y 6 horas. Una semana después ( $2^{a}$ fase) ingirieron los 82 gramos de aceite de oliva más 50 gramos de sacha inchi.

Los resultados mostraron un aumento progresivo -sin tomar el Sacha inchi- de triglicéridos hasta la toma de las 4 y media horas para disminuir a las 6 horas (1 $1^{\underline{a}}$ fase). En la curva de las tomas de sangre- consumiendo Sacha inchi, el pico se ubicó a las 3 horas para disminuir a las $41 / 2$ horas.

En la segunda fase los niveles de la curva de tolerancia a triglicéridos, tomando la plukenetia volubilis, fueron menores que en la primera fase siendo la disminución significativa $(\mathrm{p}<0.05)$ en los valores de triglicéridos a la $11 / 2$ horas y 4 1/2 horas.

Se concluyó que la plukenetia voluvilis linneo tenía un efecto significativo sobre la trigliceridemia posprandial en sujetos jóvenes. ${ }^{7}$

En un tercer estudio efecto del aceite de sacha inchi (plukenetia Volubilis Linneo) sobre el perfil lipídico en pacientes con hiperlipoproteinemia se estudió los efectos y la dosis efectiva del aceite de sacha inchi sobre el perfil lipídico de pacientes con hipercolesterolemia. Se estudió a 24 pacientes con hipercolesterolemia de tipo IIa o IIb según la clasificación de Frederickson. Se los dividió en 2 grupos cada uno de 12 pacientes, administrando al grupo A, $5 \mathrm{ml}$ de aceite de sacha inchi (2 g. de omega 3) y al grupo B, $10 \mathrm{ml}$ del aceite (4 g. de omega 3) antes del almuerzo. En ayuno de 12 horas se determinaron los niveles sanguíneos de colesterol total, fracción C-HDL, triglicéridos, glucosa, insulina y ácidos grasos no esterificados. Además se determinaron las fracciones VLDL y LDL-C. Se hizo controles de las mismas variables al mes, dos meses y cuatro meses de tratamiento contínuo. Todos los pacientes recibieron indicaciones dietéticas para limitar la ingesta de grasas insaturadas. La edad de los pacientes estuvo en el rango de 35 a 75 años con un IMC de 26.9+-3.38. Diez fueron hombres y catorce mujeres.

Con la dosis de $5 \mathrm{ml}$ del aceite (2 $\mathrm{g}$. de omega 3) hubo una significativa disminución de los niveles sanguíneos de colesterol total, fracción LDL.C, VLDL, triglicéridos y ácidos grasos no esterificados (AGNE) a la vez que se incrementó las concentraciones de HDL-C. No hubo cambios 
en la glucemia, niveles de insulina ni en el índice HOMA.

Con la dosis de $10 \mathrm{ml}$ del aceite $(4 \mathrm{~g}$. de omega 3) si existió disminución significativa de todos estos parámetros en sangre a los 4 meses de tratamiento. No se encontró disminución significativa de triglicéridos ni de la fracción VLDL, con una elevación ligera de glucosa, insulina y del índice HOMA. Al cuarto mes de tratamiento los marcadores bioquímicos se encontraban más bajos que en los controles basales con excepción del HDL-C que continuó aumentando y los AGNE que siguieron disminuyendo.

Las concentraciones promedio de los marcadores no variaron en cuanto a las dosis ni hubo cambios en el peso, medidas de cintura abdominal o cifras de tensión arterial al compararlos con los valores basales. ${ }^{8}$

El $4^{\circ}$ estudio efecto del aceite plukenetia volubilis (sacha inchi) sobre la hipercolesterolemia e hipertrigliceridemia en pacientes con hiperlipemia mixta del CAMEC- Iquitos 2009, reunió a 26 pacientes que se dividieron en 2 grupos, a uno (grupo experimental) se le administró 20 mililitros diarios de aceite sacha inchi, durante 12 semanas, mientras que el grupo control no recibió el aceite. A Ambos grupos se les administró una dieta hipolipídica y se midió colesterol total, triglicéridos y peso corporal en condiciones basales.

En los resultados se encontró una reducción de cifras de colesterol de $26.6 \%$ y de triglicéridos de $31 \%$ en el grupo experimental, mientras que en el grupo control la reducción en colesterol fue de $6.4 \%$ y de triglicéridos de $9.38 \%$ con una P de $<0.01$. No hubo diferencias entre los 2 grupos respecto al peso corporal.

Los pacientes fueron referidos del CAMEC/ Iquitos al Instituto de Medicina Tradicional ( IMT). El estudio titulado: Tratamiento alternativo de las dislipidemias con aceite de maní silvestre -sacha inchi- en el personal administrativo de la Universidad técnica del norte de la ciudad de Ibarra. Investigó el efecto de administrar $5 \mathrm{ml}$ por día de aceite sacha inchi a una población de 37 personas (19 hombres y 18 mujeres) con edades comprendidas entre los 24 y 64 años, durante un tiempo de 3 meses. Se realizó determinaciones basales en sangre de colesterol, triglicéridos, colesterol HDL, y colesterol LDL, y fueron comparados con los valores al final del estudio.

Se encontró que la media de colesterol antes de la administración fue de $248.5 \mathrm{mg} / \mathrm{dl}$ y después de la misma fue de $195.3 \mathrm{mg} / \mathrm{dl}$; de triglicéridos, antes del tratamiento, $175.3 \mathrm{mg} / \mathrm{dl}$ y después del mismo, $155.3 \mathrm{mg} / \mathrm{dl}$, de colesterol HDL antes $54.4 \mathrm{mg} / \mathrm{dl}$ y después $48.4 \mathrm{mg} / \mathrm{dl}$, y de colesterol LDL la media antes del tratamiento $136 \mathrm{mg} / \mathrm{dl} \mathrm{y}$ después $117.9 \mathrm{mg} / \mathrm{dl}$.

El estudio concluyó que las reducciones en los lípidos logradas con el aceite sacha inchi, eran significativas y recomedaron sus autores el uso de este aceite como tratamiento complementario al farmacológico en las dislipidemias. ${ }^{9}$

\section{CONCLUSIONES}

- La administración de aceite de sacha inchi proporciona un equilibrio adecuado entre los ácidos omega 6- cuyo consumo en cantidades elevadas es perjudicial- y los omega 3 que son beneficiosos para el organismo humano (omega $3,5.5 \mathrm{~g}$. por porción contra omega $6,4.5 \mathrm{~g}$ ).

- El aceite sacha inchi contiene mayor cantidad de omega 3 que el pescado.

- Con el consumo de sacha inchi se consigue en seres humanos una significativa disminución de los niveles plasmáticos de colesterol, triglicéridos y C-LDL.

- El aceite de sacha inchi reduce los niveles de triglicéridos en proporción similar al gemfibrozilo, efecto probado en animales de experimentación. Esto es importante por que el uso de $1200 \mathrm{mg}$ al dia (dosis recomendada) de gemfibrozilo en pacientes con litiasis vesicular está contraindicada. Así mismo debe tenerse precaución con la administración conjunta con anticoagulantes.

- Las limitaciones de estos estudios estarían constituídas por el reducido número de los sujetos de las investigaciones, siendo deseable investigar los efectos de la plukenetia Volubilis en un universo mayor de pacientes dislipémicos y también con intolerancia a la glucosa.

\section{REFERENCIAS BIBLIOGRÁFICAS}

1. Tama Viteri. F. A, Clínica y terapéutica de las dislipidemias. 1를 Edición. Editorial Universidad de Guayaquil, 2011.

2. Rodríguez García. J.L, Diagnóstico y tratamiento médico. Marbán libros. 2013. 
3. Gurfinkel. E. P, La enfermedad coronaria. $1^{\underline{a}}$ Ed, Buenos Aires: Journal 2008.

4. Ganong. W. F, Fisiología Médica. 19ํㅗㄹ ed. México, Editorial El manual Moderno, 2004.

5. González Gustavo. F, González. Carla, Estudio de seguridad y tolerancia en sujetos normales que reciben aceite de sacha inchi (Plukenetia Volubilis). Universidad peruana Cayetano Heredia, Octubre 2013.

6. Vicuña Ríos. A, Izquierdo Henríquez. E, Huamán Saavedra .J. J, Gemfibrozilo versus aceite de Sacha Inchi en la reducción de niveles de triglicéridos séricos en Rattus rattus var albinus. Acta Med Per, 29 (2). 2012.

7. Huamán J, Chávez K, Castañeda E, Carranza. S, Chávez. T, Beltrán. Y, Caffo. C, Cadillo. R, Cadenillas. J, Efecto de la Plukenetia Volubilis Linneo (sacha inchi) en la trigliceridemia postprandial. An. Fac. Med, Vol 69, No 4, Lima, Dic 2008.

8. Garmendia F, Pando R, Ronceros G, Efecto del aceite de Sacha Inchi (Plukenetia volúbilis) sobre el perfil lipídico en pacientes con hiperlipoproteinemia. Informe preliminar presentado en el VI Congreso Internacional de la Asociación Peruana de estudio de la obesidad y aterosclerosis, Lima, Julio 2010.

9. Jácome Pozo. M. V, Laboratorio clínico, Universidad técnica del Norte.

10. Aranda Ventura. J.A, Efecto del aceite de plukenetia Volubilis (Sacha inchi) sobre la hipercolesterolemia e hipertrigliceridemia en pacientes con hiperlipemia mixta del CAMEC-Iquitos 2009. 